\title{
INFLUÊNCIA DA COMPOSIÇÃO DA SALMOURA SOBRE OS PARÂMETROS FÍSICO-SENSORIAIS E MICROBIOLÓGICOS DE FILÉS DE PEITO DE FRANGO MARINADOS POR IMERSÃO
}

\author{
ANNA CLAUDIA SIMAS PORTO * \\ REGINA COELI DE OLIVEIRA TÔRRES * \\ EUNICE CASSANEGO ILHA * \\ MARILDE TEREZINHA BORDIGNON LUIZ ** \\ ERNANI SEBASTIÃO SANT'ANNA ***
}

\begin{abstract}
Avaliou-se a influência da composição da salmoura sobre os parâmetros físico-sensoriais e microbiológicos de filés de peito de frango marinados por imersão. O experimento foi constituído por cinco formulações de salmoura. A proporção salmoura:frango foi de 2:1 (v/p). $\mathrm{O}$ processo de marinação ocorreu a temperatura de $5{ }^{\circ} \mathrm{C} / 12$ horas, seguido de drenagem por 30 minutos. As amostras destinadas ao acompanhamento da vida de prateleira, 6 e 11 dias, foram armazenadas a $5^{\circ} \mathrm{C}$. Foram avaliados ganho de peso durante a marinação $e$ perda de peso no cozimento, sendo efetuadas determinação de $\mathrm{pH}$, contagem de microrganismos aeróbios psicrotróficos, contagem de Staphylococcus aureus e pesquisa de Salmonella sp. Os menores ganhos de peso foram verificados para os tratamentos com adição de vinagre na salmoura, fato atribuído à redução do pH próximo ao ponto isoelétrico da carne. Estes tratamentos evidenciaram as maiores perdas de peso durante o cozimento. A matéria-prima apresentou ausência de Salmonella sp. e contagens de Staphylococcus aureus e microrganismos psicrotróficos dentro dos limites estabelecidos pelo ICMSF. Após 11 dias de armazenamento, apenas os tratamentos com vinagre na composição da salmoura apresentaram contagem de microrganismos aeróbios psicrotróficos dentro do limite estabelecido pelo ICMSF $\left(10^{7} \mathrm{UFC} / \mathrm{g}\right)$. As melhores texturas foram verificadas nos tratamentos com adição de alecrim na composição da salmoura.
\end{abstract}

* M.Sc. em Ciência dos Alimentos, Departamento de Ciência e Tecnologia de Alimentos, Universidade Federal de Santa Catarina (UFSC), Florianópolis, SC.

** Professor Adjunto, Departamento de Ciência e Tecnologia de Alimentos, UFSC.

*** Professor Titular, Departamento de Ciência e Tecnologia de Alimentos, UFSC. (e-mail: ernanis@cca.ufsc.br). 


\section{INTRODUÇÃO}

O consumo de carnes semi-preparadas vem crescendo de maneira significativa nos grandes centros urbanos, devido a mudança no estilo de vida que exige operações culinárias rápidas. Desta forma, os frigoríficos e casas de carne passaram a investir em novas tecnologias para cortes, conservação e embalagem para atender a esta demanda (12). Com esta mudança no perfil do consumidor, o mercado de cortes tornou-se opção mais rentável que o de frangos inteiros, oferecendo hoje quinze diferentes cortes. Este mercado de pouca expressão fabril cresceu em importância, tamanho e tecnologia (11).

Os marinados surgiram como alternativa de produto semi-preparado com tempo de conservação prolongado, ampliando as opções de consumo para carnes e aves. Podem ser designados como carnes, aves e peixes, cuja penetração ou difusão da salmoura, geralmente elaborada com vinagre e especiarias, ocasiona melhoria na textura e no sabor.

A interação química proteína-água, que ocorre no processo de marinação, deve-se à atração das cargas que são afetadas pelas condições do meio, como $\mathrm{pH}$. Com a diminuição do $\mathrm{pH}$ do meio, o valor obtido pode aproximarse do ponto isoelétrico ( $\mathrm{pl}$ ) das proteínas, restringindo as cargas disponíveis, diminuindo sensivelmente as interações proteína-água e aumentando as interações proteína-proteína (8).

Fosfatos alcalinos aumentam o pH do tecido muscular, afastando as proteínas do seu pl, além disso, sequestram ou atraem os íons de metais carregados negativamente, deixando as cargas positivas disponíveis para se ligarem a molécula da água (8).

A adição de sal ao produto realça o sabor da carne e atua como agente antimicrobiano e higroscópico, contribuindo também para a retenção das moléculas de água (8). FRONING \& SACKETT (5) em suas pesquisas demonstraram o efeito sinergístico do sal e fosfatos na carne, ou seja, proporciona maior rendimento, amplia a capacidade de retenção de água e melhora consideravelmente a textura.

Os ácidos acético e láctico são usados no controle de deterioração de carnes e organismos patogênicos. Especiarias são, geralmente, usadas em alimentos como agentes flavorizantes e amaciantes, além de atuarem como agentes preservativos, pela presença de óleos essenciais e oleoresinas (16). Diversas especiarias e ervas apresentam atividade antimicrobiana, devido muitas vezes a presença de um componente particular (6). Embora esta atividade represente promissora área de pesquisa, a quantidade de condimentos normalmente utilizada como flavorizante pode não ser suficiente para exercer efeito antimicrobiano (15). Trabalhos conduzidos sobre especiarias como orégano, alecrim e sálvia 
indicam que a atividade antimicrobiana deve-se a presença de compostos fenólicos (3).

O presente trabalho teve por objetivo avaliar a influência da adição de tripolifosfato de sódio e variação de $\mathrm{pH}$, no ganho de peso, perda de peso no cozimento e textura de filés de peito de frango marinados por imersão. Avaliou-se ainda a influência da adição de vinagre e alecrim (Rosmarinus officinalis) na qualidade microbiológica dos filés armazenados a $5{ }^{\circ} \mathrm{C}$.

\section{MATERIAL E MÉTODOS}

\subsection{MATÉRIA-PRIMA}

Foram utilizados cortes de filés (peso médio de $130 \mathrm{~g}$ ) de peito de frango, resfriados a $5{ }^{\circ} \mathrm{C}$, adquiridos no mercado local. A matéria-prima foi transportada em caixas térmicas até o Laboratório de Biotecnologia Alimentar da Universidade Federal de Santa Catarina.

\subsection{PREPARO DAS SALMOURAS}

O experimento, em triplicata, foi constituído por cinco formulações de salmoura, com água destilada fervida e resfriada a $5^{\circ} \mathrm{C}$, correspondendo aos seguintes tratamentos: controle $=4 \%$ de cloreto de sódio; tratamento $\mathrm{A}$ $=4 \%$ de cloreto de sódio e $2 \%$ de tripolifosfato de sódio; tratamento $\mathrm{B}=$ $4 \%$ de cloreto de sódio, $2 \%$ de tripolifosfato de sódio e $0,1 \%$ (p/v) de folhas de alecrim in natura maceradas; tratamento $\mathrm{C}=4 \%$ de cloreto de sódio, $2 \%$ de tripolifosfato de sódio e vinagre até redução do $\mathrm{pH}$ para 5,0 ; tratamento $\mathrm{D}=4 \%$ de cloreto de sódio, $2 \%$ de tripolifosfato de sódio, $0,1 \%$ $(\mathrm{p} / \mathrm{v})$ de folhas de alecrim in natura maceradas e vinagre até redução do $\mathrm{pH}$ para 5,0 .

A proporção salmoura:frango, para todos os tratamentos, foi de 2:1 (v/p).

\subsection{MARINAÇÃO}

O processo de marinação por imersão ocorreu a temperatura de $5^{\circ} \mathrm{C}$, por 12 horas (parâmetros obtidos em ensaios preliminares). Os filés, pesados individualmente em sacos de polietileno, foram submetidos aos tratamentos (controle, A, B, C e D). Em seguida realizou-se a drenagem por 30 minutos, em temperatura de $5^{\circ} \mathrm{C}$, com auxílio de peneiras, sendo os filés transferidos para sacos de polietileno estéreis e pesados para avaliação do ganho de peso (\%). As amostras destinadas ao acompanhamento da vida de prateleira, 6 e 11 dias, foram armazenadas a $5{ }^{\circ} \mathrm{C}$. 


\subsection{ANÁLISES FÍSICO-QUÍMICAS}

\subsubsection{Ganho de peso}

O ganho de peso foi avaliado como água absorvida após o processo de marinação (peso drenado) e calculado como:

Ganho de peso $=100 \times($ peso drenado - peso inicial $) /$ peso inicial .

\subsubsection{Perda de peso}

Os filés de peito foram assados em chapa elétrica $\left(220{ }^{\circ} \mathrm{C}\right)$ até a temperatura interna no centro do filé atingir $70^{\circ} \mathrm{C}$, em seguida foram pesados e a perda de peso calculada como:

Perda de peso $=100 \times$ (peso drenado - peso cozimento $) /$ peso drenado .

\subsubsection{Determinação de $\mathrm{pH}$}

Utilizou-se pHmetro para determinar o pH em $10 \mathrm{~g}$ da amostra, diluída com $100 \mathrm{~mL}$ de água destilada, de acordo com TERRA \& BRUM (13).

\subsection{ANÁLISES MICROBIOLÓGICAS}

Procedeu-se a contagem de microrganismos aeróbios psicrotróficos (semeadura em superfície, em ágar para contagem padrão - PCA e as placas incubadas a $7 \stackrel{\circ}{\circ}$ por 10 dias); a contagem de Staphylococcus aureus e pesquisa de Salmonella sp. (1), na matéria-prima e nos filés marinados, nos tempos 0,6 e 11 dias de armazenamento a $5^{\circ} \mathrm{C}$. O tempo de armazenamento foi estabelecido considerando que a vida útil de cortes de frango e frango inteiro resfriado é de 5-6 e 10-12 dias, respectivamente.

\subsection{AVALIAÇÃO SENSORIAL}

Os files assados foram cortados em cubos de $2 \mathrm{~cm}^{2}$ e submetidos a avaliação sensorial, por painel treinado, composto por seis julgadores, mediante teste de escala hedônica de 5 pontos ( 1 - péssimo e 5 excelente), para o atributo textura.

\subsubsection{Análise estatística}

Utilizou-se o delineamento experimental inteiramente casualizado, com três repetições de cada tratamento. Foi aplicada a análise de variância para 
verificar diferenças significativas entre os tratamentos para o atributo textura e depois de comprovação o Teste de Tukey a 5\%. Os cálculos foram efetuados pelo programa estatístico Statgraphics (Statistical Graphics System, versão 7.0) (7).

\section{RESULTADOS E DISCUSSÃO}

O maior ganho de peso foi verificado nos tratamentos B $(8,62 \%)$ e A $(8,60 \%)$. Estes valores podem ser atribuídos à adição de tripolifosfato na salmoura o que contribuiu para o aumento da força iônica do meio, aumentando a capacidade de absorção de água (efeito salting in).

De acordo com WONG (14) a adição de fosfatos em carnes reduz a perda de água de constituição pelo incremento da ligação de água ou retenção do suco natural. Isto ocorre pelo aumento do $\mathrm{pH}$ e da força iônica e por complexarem o magnésio e o cálcio das ligações protéicas, assim a actomiosina pode dissociar-se expondo mais sítios de ligação e aumentando o espaço estrutural da miofibrila para hidratação.

Nos tratamentos D e C ocorreram os menores ganhos de peso, $5,47 \%$ e $5,23 \%$, respectivamente. Tal fato pode ser atribuído a adição de vinagre na salmoura, que reduziu o pH da carne (5,72 e 5,65, respectivamente) próximo ao ponto isoelétrico das proteínas miofibrilares da carne de frango $(\mathrm{pH} 5,5)$. Foi observado que no pl as cargas das proteínas são nulas e a capacidade de hidratação é mínima, ocasionando diminuição na capacidade de hidratação da actina e miosina e aumento da atração proteína-proteína.

O tratamento Controle apresentou ganho de peso intermediário $(6,73 \%)$ em relação aos demais tratamentos, o que pode ter ocorrido pela ausência do efeito tampão conferido pelo tripolifosfato de sódio (Tabela 1).

As maiores perdas de peso foram observadas nos tratamentos Controle, $\mathrm{D}$ e C (Tabela 1). Analisando os valores de $\mathrm{pH}$ da carne, no tempo zero, verifica-se que estes encontraram-se mais próximos do $\mathrm{pl}$ da carne de frango.

A legislação federal (2) estabelece para aves resfriadas ausência de Salmonella sp. em $25 \mathrm{~g}$. A pesquisa de Staphylococcus aureus fundamentou-se no fato deste microrganismo estar envolvido em inúmeros casos de toxinfecção alimentar.

Os resultados das análises microbiológicas da matéria-prima (Tabela 2) apresentaram-se dentro dos limites aceitáveis de qualidade. 


\section{TABELA 1 - VALORES ${ }^{1}$ DE PH DA SALMOURA, DOS FILÉS ${ }^{2}$ DE PEITO DE FRANGO MARINADOS POR IMERSÃO (TEMPO 0) E ARMAZENADOS A 5 ㅇ (TEMPO 6 E 11 DIAS), GANHO DE PESO APÓS MARINAÇÃO E PERDA DE PESO NO COZIMENTO}

\begin{tabular}{c|cc|ccc|cc}
\hline Tratamentos & \multicolumn{2}{|c|}{$\mathrm{pH} \mathrm{da} \mathrm{Salmoura}$} & \multicolumn{2}{c|}{$\mathrm{pH}$ dos filés } & (Tempo $^{3}$ & \multicolumn{2}{c}{ Perda de peso } \\
\cline { 2 - 8 } & Inicial $^{4}$ & Final $^{5}$ & 0 & 6 & 11 & $(\%)$ & $(\%)$ \\
\hline Controle & 7,20 & 5,73 & 5,86 & 5,78 & 6,41 & 6,73 & 27,65 \\
A & 7,29 & 6,38 & 6,43 & 6,23 & 6,57 & 8,60 & 23,17 \\
B & 7,29 & 6,41 & 6,29 & 6,09 & 6,44 & 8,62 & 24,51 \\
C & 5,00 & 5,36 & 5,65 & 5,68 & 5,67 & 5,23 & 26,08 \\
D & 5,00 & 5,36 & 5,72 & 5,80 & 5,88 & 5,47 & 27,69 \\
\hline
\end{tabular}

${ }^{1}$ Média de três repetições.

${ }^{2} \mathrm{pH}$ da matéria-prima $=6,0$.

3 Tempo de armazenamento em dias.

${ }_{5}^{4} \mathrm{pH}$ antes da marinação.

${ }^{5} \mathrm{pH}$ da salmoura após 12 horas de marinação a $5{ }^{\circ} \mathrm{C}$.

Tratamentos: Controle ( $4 \%$ de cloreto de sódio); A ( $4 \%$ de cloreto de sódio e $2 \%$ de tripolifosfato de sódio); B ( $4 \%$ de cloreto de sódio, $2 \%$ de tripolifosfato de sódio e $0,1 \%$ $\mathrm{p} / \mathrm{v}$ de folhas de alecrim maceradas); C (4\% de cloreto de sódio, $2 \%$ de tripolifosfato de sódio e vinagre até redução do pH para 5,0$)$ e $\mathrm{D}(4 \%$ de cloreto de sódio, $2 \%$ de tripolifosfato de sódio, $0,1 \% \mathrm{p} / \mathrm{v}$ de folhas de alecrim maceradas e vinagre até redução do $\mathrm{pH}$ para 5,0$)$.

Todos as amostras apresentaram ausência de Salmonella sp. em $25 \mathrm{~g}$ durante os tempos 0 (Tabela 2), 6 e 11 dias (Tabela 3) de armazenagem. Os resultados obtidos para Staphylococcus aureus foram $<10^{2} \mathrm{UFC} / \mathrm{g}$ para todos os tratamentos nos tempos 0 (Tabela 2), 6 e 11 dias de armazenamento (Tabela 3).

Embora a contagem de microrganismos aeróbios psicrotróficos indiquem o grau de deterioração de alimentos refrigerados, a legislação brasileira não estabelece padrão para estes microrganismos. Entretanto, a International Commission on Microbiological Specificacions for Foods (9) estabelece $10^{6}$ a $10^{7} \mathrm{UFC} / \mathrm{g}$ como padrão. De acordo com FRANCO \& LANDGRAF (4) o produto que apresenta contagens nesta faixa, já demonstra qualidade marginal.

De acordo com a Tabela 3, no sexto dia de armazenamento, a contagem de microrganismos aeróbios psicrotóficos nos tratamentos $\mathrm{C}$ e $\mathrm{D}$ mostrouse foi inferior $\left(10^{5} \mathrm{UFC} / \mathrm{g}\right)$ aos limites do ICMSF (9), enquanto os demais tratamentos já demonstravam qualidade marginal ( $\left.10^{7} \mathrm{UFC} / \mathrm{g}\right)$.

No décimo primeiro dia de armazenamento, apenas os tratamentos $C$ e $D$ encontravam-se no limite proposto pelo ICMSF (9). Tal fato pode ser 
atribuído à redução do $\mathrm{pH}$ pela adição de vinagre, com a conseqüente inibição da microbiota psicrotrófica.

TABELA 2 - ANÁLISES MICROBIOLÓGICAS DOS FILÉS DE PEITO DE FRANGO IN NATURA (MATÉRIA-PRIMA) E SUBMETIDOS AOS DIFERENTES TRATAMENTOS, NO TEMPO ZERO

\begin{tabular}{l|c|c|c}
\hline Amostra & Salmonella sp. (25 g) & S. aureus (UFC/g) & $\begin{array}{c}\text { Psicrotróficos } \\
\text { (UFC/g) }\end{array}$ \\
\hline Matéria-prima & ausente & $<10^{2}$ & $5,4 \times 10^{4}$ \\
Controle & ausente & $<10^{2}$ & $1,4 \times 10^{4}$ \\
A & ausente & $<10^{2}$ & $2,3 \times 10^{4}$ \\
B & ausente & $<10^{2}$ & $1,6 \times 10^{4}$ \\
C & ausente & $<10^{2}$ & $6,1 \times 10^{3}$ \\
D & ausente & $<10^{2}$ & $5,3 \times 10^{3}$ \\
\hline
\end{tabular}

Em relação às características organoléticas da carne, os filés de frango não apresentaram odores fétidos nem limosidade, geralmente associados com carnes em deterioração, em nenhum dos períodos de armazenamento (Tabela 3). Entretanto, as contagens de microrganismos aeróbios psicrotróficos para os tratamentos $\mathrm{A}$ e $\mathrm{B}$, a partir do $6^{\circ}$ dia, já haviam atingido os limites estabelecidos pelo ICMSF (10 $\mathrm{UFC} / \mathrm{g})$.

\section{TABELA 3 - ANÁLISES MICROBIOLÓGICAS DOS FILÉS DE PEITO DE FRANGO MARINADOS, SUBMETIDOS A DIFERENTES TRATAMENTOS, ARMAZENADOS A $5 \stackrel{\circ}{\circ} \mathrm{C}$, DURANTE 6 E 11 DIAS}

\begin{tabular}{c|c|c|c}
\hline Amostra/dias & Salmonella sp. (25 g) & S. aureus (UFC/g) & $\begin{array}{c}\text { Psicrotróficos } \\
\text { (UFC/g) }\end{array}$ \\
\hline Controle/6 & ausente & $<10^{2}$ & $1,0 \times 10^{7}$ \\
A/6 & ausente & $<10^{2}$ & $1,2 \times 10^{7}$ \\
B/6 & ausente & $<10^{2}$ & $1,4 \times 10^{7}$ \\
C/6 & ausente & $<10^{2}$ & $3,1 \times 10^{5}$ \\
D/6 & ausente & $<10^{2}$ & $2,5 \times 10^{5}$ \\
\hline Controle/11 & ausente & $<10^{2}$ & $5,0 \times 10^{9}$ \\
A/11 & ausente & $<10^{2}$ & $2,8 \times 10^{9}$ \\
B/11 & ausente & $<10^{2}$ & $9,8 \times 10^{8}$ \\
C/11 & ausente & $<10^{2}$ & $1,5 \times 10^{7}$ \\
D/11 & ausente & $<10^{2}$ & $1,2 \times 10^{7}$ \\
\hline
\end{tabular}

Para o atributo textura (tempo zero) (Tabela 4) houve diferença significativa $(p<0,05)$ apenas entre os tratamentos B e C. Entretanto, as maiores médias foram atribuídas aos tratamentos $B(2,70)$ e $D(2,65)$, adicionados de alecrim, que por sua vez pode conter algum constituinte capaz de 
interferir na maciez da carne. Segundo LEE \& ASHMORE (10) a presença de enzimas proteolíticas, em especiarias como o gengibre, são responsáveis pelo amaciamento de carnes.

\title{
TABELA 4 - MÉdIAS DAS NOTAS ATRIBUÍDAS, PELO TESTE DE ESCALA HEDÔNICA, ATRIBUTO TEXTURA, PARA OS FILÉS DE FRANGO MARINADOS POR IMERSẪO
}

\begin{tabular}{l|l}
\hline Amostra & Médias* \\
\hline B & $2,70^{\mathrm{a}}$ \\
D & $2,65{ }^{\mathrm{ab}}$ \\
A & $2,42 \mathrm{ab}$ \\
Controle & $2,36{ }^{\mathrm{ab}}$ \\
C & $2,28{ }^{\mathrm{b}}$ \\
\hline
\end{tabular}

* Médias assinaladas com a mesma letra não diferem estatisticamente entre si, pelo teste de Tukey ao nível de $5 \%$ de probabilidade.

\section{CONCLUSÃO}

A adição de tripolifosfato de sódio na salmoura aumentou a absorção de água, proporcionando aumento no rendimento.

Apenas os filés de peito de frango marinados com salmoura adicionada de vinagre apresentaram-se de acordo com os padrões de qualidade no sexto dia de armazenamento.

Em relação à textura, as melhores médias foram atribuídas aos tratamentos adicionados de alecrim.

\begin{abstract}
It was evaluated the influence of brine composition on physicosensory and microbiological parameters in breast fillet of marinated chicken by immersion. The experiment was constituted by five brine formulations. The proportion of brine:fillet was 2:1 ( $/ / \mathrm{w})$ for all treatments. The marination process occurred at $5^{\circ} \mathrm{C} / 12$ hours, following of drainage for 30 minutes. The samples were stored at $5{ }^{\circ} \mathrm{C}$ at 6 and 11 days to monitor shelf-life. It was then evaluated weight gain and loss, $\mathrm{pH}$ determination, count of psicrotrophics microorganisms and Staphylococcus aureus and absence of Salmonella sp. The lowest values of weight gain were observed in treatments with addition of vinegar in brine, probably due to the decrease of $\mathrm{pH}$ close to the meat isoelectric point. This treatments also evidenced the highest weight loss during the cooking. The raw material presented absence of Salmonella sp. and a count of Staphylococcus aureus and psicrotrophics microorganisms within quality limits established by ICMSF. After 11 days of storage, the treatments with vinegar in the brine composition presented count of psicrotrophics microorganisms within limits established by ICMSF $\left(10^{7} \mathrm{CFU} / \mathrm{g}\right)$. The best textures were presented in treatments with addition of rosemary (Rosmarinus officinalis) in brine composition.
\end{abstract}




\section{REFERÊNCIAS}

1 AMERICAN PUBLIC HEALTH ASSOCIATION. Compendium of methods for the microbiological examination of food. $3^{\text {rd }}$. ed. Washington: APHA, 1992. $1219 \mathrm{p}$.

2 BRASIL. Ministério da Saúde. Secretaria de Vigilância Sanitária. Portaria n. 451 de 19 set. 1997. Aprova o regulamento técnico, princípios gerais para o estabelecimento de critérios e padrões microbiológicos para alimentos. Diário Oficial [da] República Federativa do Brasil, Brasília, n. 124-E, p. 4, 2 de jul. de 1998. Seção I.

3 BROOKMAN, P. Antioxidants and consumer acceptance. Food Technology, v. 45, n. 10, p. 24-28, 1991.

4 FRANCO, B.D.G.M.; LANDGRAF, M. Microbiologia dos alimentos. São Paulo: Atheneu, 1996. 182 p.

5 FRONING, G. W.; SACKETT, B. Effect of salt and phosphates during tumbling of turkey breast muscle on meat characteristics. Journal of Poultry Sience, v. 64, p. 1328-1333, 1985.

6 GIESE, J. Spices and seasonings blends: a taste for all seasons. Food Technology, v. 48, n. 4, p. 88-98, 1994.

7 GRAPHIC SOFTWARE SYSTEMS. Statgraphics: statistical graphics system. Cary, USA, 1985-93. Version 7.0.

8 GUAJARDO, E. R.; JARAMILLO, H.G. El marinado: una opción rentable en la carne de bovino. Carnetec, v. 2, n. 5, p. 20-24, 1995.

9 INTERNATIONAL COMMISSION ON MICROBIOLOGICAL SPECIFICATIONS FOR FOODS. Microorganisms in foods: their significance and methods of enumeration. Toronto: University of Toronto, 1978.

10 LEE, Y.B.; KIM, Y.S.; ASHMORE, C.R. Antioxidant property in ginger rhizome and the application to meat products. Journal of Food Science, v. 51, n. 1, p. 20-23, 1986

11 NUNES, F. G. Processamento avícola: dinamismo e sucesso. Avicultura Industrial, v. 86, n. 1037, p. 25-28, 1996.

12 O SETOR da carne no Brasil. Revista Nacional da Carne, v. 20, n. 231, p. 72-73, 1996.

13 TERRA, N. N.; BRUM, M. A. R. Carnes e seus derivados: técnicas de controle de qualidade. São Paulo: Nobel, 1988. 121 p. 
14 WONG, D.W.S. Mechanisms and theory in chemistry. New York: Van Nostrand Reinhold, 1989. 314 p.

15 ZAIKA, L.L. Spices and herbs: their antimicrobial activity and its determination. Journal of Food Safety, n. 9, p. 97-118, 1988.

16 ZIAUDDIN, K. S.; RAO, H. S.; FAIROZE, N. Effects of organic acids and spices on quality and shelf-life of meats at ambient temperature. Journal of Food Technology, v. 33, n. 3, p. 255-258, 1996. 